\title{
Automatic Defect Categorization Based On Fault Triggering Conditions
}

\author{
Xin Xia*‡, David $\mathrm{Lo}^{\dagger}$, Xinyu Wang*, and Bo Zhou*§ \\ ${ }^{*}$ College of Computer Science and Technology, Zhejiang University, China \\ ${ }^{\dagger}$ School of Information Systems, Singapore Management University, Singapore \\ xxkidd@zju.edu.cn, davidlo@smu.edu.sg, \{xinyuwang, bzhou\}@zju.edu.cn
}

\begin{abstract}
Due to the complexity of software systems, defects are inevitable. Understanding the types of defects could help developers to adopt measures in current and future software releases. In practice, developers often categorize defects into various types. One common categorization is based on fault triggers of defects. Fault trigger is a set of conditions which activate a defect (i.e., fault) and propagate the defect into a failure. In general, there are two types of defect based fault triggering conditions, Bohrbug and Mandelbug. Bohrbug refers to a bug which can be easily isolated, and its activation and error propagation is simple. Mandelbug refers to a bug whose activation and/or error propagation is complex (e.g., a time lag between the fault activation and the failure occurrence). With these category labels, developers can better perform post-mortem analysis to identify common characteristic of the defects, and design specific fault-tolerance mechanisms.
\end{abstract}

However, in most software systems, these category labels are often unavailable. To address this problem, in this paper, we propose a text mining solution which categorize defects into fault trigger categories by analyzing the natural-language description of bug reports. A previous study shows that Mandelbug is more complex and needs more time to be fixed. Thus, to better identify Mandelbugs, we propose a novel fUzzy Set based fEature Selection algorithm named USES, which selects the features (i.e., terms) which have high ability to distinguish Mandelbugs from Bohrbugs. USES first caches a set of terms based on their fuzzy affinity scores to Bohrbug or Mandelbug. Next, it iterates many times, and in each iteration, it selects a subset of terms, and builds a classifier on these terms. USES selects the classifier and the terms which could achieve the best performance on a training data. We evaluate our solution on 4 datasets including Linux, Mysql, Apache HTTPD, and AXIS containing a total of 809 bug reports. We show that USES with naive Bayes multinomial achieves the best performance; it achieves Mandelbug F-measure scores of $0.298-0.615$. We also compare USES with other baseline approaches. The results show that USES on average improves Mandelbug F-measure scores of the best performing baseline by $12.3 \%$.

Keywords-Fault Triggers, Bohrbug, Mandelbug, Feature Selection, Fuzzy Set, Categorization, Machine Learning

\section{INTRODUCTION}

Defects (i.e., bugs or faults) are prevalent in software systems, and appear in all stage of software development lifecycle. A previous study shows that the cost of debugging in a software system consumes $50 \%-80 \%$ of the development and maintenance cost [1]. There are various kinds of defects. Some

\footnotetext{
${ }^{\ddagger}$ The work was done while the author was visiting Singapore Managemen University.

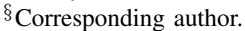

kinds of defects are easy to reproduce, and show consistent behaviors under the same inputs. Some kinds of defects are hard to reproduce, and their behaviors are inconsistent when the same inputs are given, e.g., concurrency defects [2], [3]. To ensure the reliability of software systems, the management of defects is necessary. Understanding the types of defects could help developers to perform post-mortem analysis, and adopt corresponding measures, such as adding more human resource, refactoring, and developing an automated defect detection tool, to prevent the recurrence of defects in future software releases.

One common categorization is based on fault triggers of defects. Fault trigger is a set of conditions which activate a defect (i.e., fault) and propagate the defect into a failure. In some cases, fault triggers are complex, which would cause the failures become extremely hard to reproduce. For example, the timing of events and interactions with other systems (e.g., operating system) [4]. Traditional dynamic testing techniques cannot easily detect these kinds of faults, due to the challenges of reproducing complex fault triggers in a test environment. In general, Grottke and Trivedi propose two categories of defects based fault triggering conditions, Bohrbug and Mandelbug [5]. Bohrbug refers to a bug which can be easily isolated, and its activation and error propagation is simple. Mandelbug refers to a bug whose activation and/or error propagation is complex (e.g., a time lag between the fault activation and the failure occurrence).

Albeit the benefits of categorizing defects into types, in most software systems, these category labels are often unavailable as such categorization potentially involves much manual effort, and the project team may not have the budget for defect categorization. Thus, there would be a need for an automated tool which could help developers in assigning categories to defects during post-mortem analysis.

In this paper, we propose a text mining solution which categorize defects into two fault trigger categories: Bohrbug and Mandelbug. Our goal is to automatically classify a defect into one of the two categories according to its natural-language description available in the corresponding bug report. Cotroneo et al. conclude that Mandelbugs need longer time to fix, and require specific strategies to be dealt with [6]. For example, in Linux, the average time to fix a Bohrbug is 157 hours, while the average time to fix a Mandelbug is 230 hours [6]. Also in some projects (e.g., apache HTTP and AXIS), the number of bugs which belongs to Mandelbug category are much less than these of Bohrbug category. Thus, we focus on identifying Mandelbugs. 
We propose a novel fUzzy Set fEature Selection algorithm named USES, which selects the features (i.e., terms) which have high ability to distinguish Mandelbugs from Bohrbugs. In more detail, USES first computes the fuzzy affinity scores for each terms to each category (i.e., Bohrbug and Mandelbug). Then, USES caches a set of terms which have the highest fuzzy affinity scores to Bohrbug or Mandelbug. Next, it iterates many times, and in each iteration, it selects a subset of terms, and builds a classifier on these terms. Also, USES evaluates the performance of each of the classifiers on a training data. Finally, the classifier and the terms which could achieve the best performance on the training data are used to predict the categories of new unlabeled defects.

We evaluate our solution on 4 datasets including Linux, Mysql, Apache HTTPD, and AXIS containing a total of 809 bug reports. ${ }^{1}$ We show that $U S E S$ with naive Bayes multinomial [7] achieves Mandelbug F-measure scores of $0.298-0.615$ which outperforms a number of baseline approaches. We compare USES with other state-of-the-art classification techniques without feature selection, e.g., naive Bayes multinomial [7], naive Bayes [7], SVM [8], logistic regression [8], and RBF network [9], and naive Bayes multinomial with information gain [8] as the feature selection technique. The results show that USES on average improves Mandelbug F-measure scores of the best baseline techniques (i.e., naive Bayes multinomial with information gain as the feature selection technique) by $12.3 \%$.

The main contributions of this paper are as follows:

1) We propose a text mining solution that automatically classifies defects into categories based on fault triggering conditions. These categories could be used for post-mortem analysis, and design fault-tolerance mechanisms.

2) Considering Mandelbugs are complex and need more time to fix, we propose a fuzzy set feature selection method USES to select important features from the natural language description of defects.

3) We perform an empirical evaluation of our automated defect categorization tool. The result based on 809 manually categorized defects shows that our tool improves a number of baseline techniques by a substantial margin.

The remainder of the paper is organized as follows. We describe the preliminary materials and motivating examples in Section II. We outline the overall framework of our defect categorization based on fault triggering conditions solution in Section III. We elaborate how the features are extracted from bug reports in Section IV. We present fuzzy set feature selection method USES in Section V. We report the experiment results in Section VI. We present the threats to validity of our paper in Section VII. We describe related work in Section VIII. We conclude and mention future work in Section IX.

\section{PReliminaries AND Motivating Example}

In this section, we first describe preliminary materials on fault trigger categories in Section II-A. Next, we present examples which motivate the need for classifying defects into fault trigger categories in Section II-B.

\footnotetext{
${ }^{1}$ We have made the dataset publicly available from: http://goo.gl/aeKoGR
}

Axis / AXIS-408

SimpleAxisServer's 'start' doesn't return anymore

Agile Board

Description

In Beta 3 SimpleAxisServer's start method used to return (spawn a thread) Since then it was changed to be based on a doThread flag but there is no way to set this flag w/o going through main() - which some people may

not do (ie. (new SimpleAxisServer()).start()).

Fig. 1. An Example of Bohrbug in AXIS with BUGID=AXIS-1261.

\section{A. Fault Trigger Categories}

Grottke and Trivedi define two types of defects based on their fault trigging conditions, i.e., Bohrbug and Mandelbug [5]. These two categories are related to the conditions of fault activation and error propagation.

Definition 1: (Bohrbug. ) A bug (i.e., fault) which can be easily isolated and manifests consistently under a well-defined set of conditions, since its activation and error propagation lack "complexity".

Definition 2: (Mandelbug. ) A bug which is difficult to isolate, and/or the failures caused by it are hard to reproduce. Its activation and/or error propagation conditions are complex, where "complexity" can take the following two forms:

1) A time lag between fault activation and failure occurrence.

2) The activation and/or error propagation conditions depend on interactions between the conditions occurring inside the application and conditions that accrue within the system-internal environment (e.g., hardware, operating system).

According to Grottke and Trivedi, a software defect only belongs to exactly one of the above two categories [5]. Thus, these two types are complementary of each other.

\section{B. Motivating Examples}

Figure 1 shows an example of Bohrbug in AXIS with BugID=AXIS-408. ${ }^{2}$ It describes a defect that the method new SimpleAxisserver(()) . start() does not return anymore. To reproduce this defect, developers just need to call the method SimpleAxisServer()). start() in their source code.

Figure 2 shows an example of Mandelbug in AXIS with BugID=AXIS-1261. ${ }^{3}$ It describes a defect that causes a NullPointerException to be thrown out. The root cause of this defect is a race condition. Notice that this defect would be hard to reproduce, since it only happens occasionally. Thus, the defect is classified as a Mandelbug by Cotroneo et al. [6].

Observations and Implications. From the above 2 defects, we can observe the following:

1) Considering both Bohrbug and Mandelbug, we find Mandelbug would be difficult to fix, since it is hard

\footnotetext{
${ }^{2}$ https://issues.apache.org/jira/browse/AXIS-408

${ }^{3}$ https://issues.apache.org/jira/browse/AXIS-1261
} 


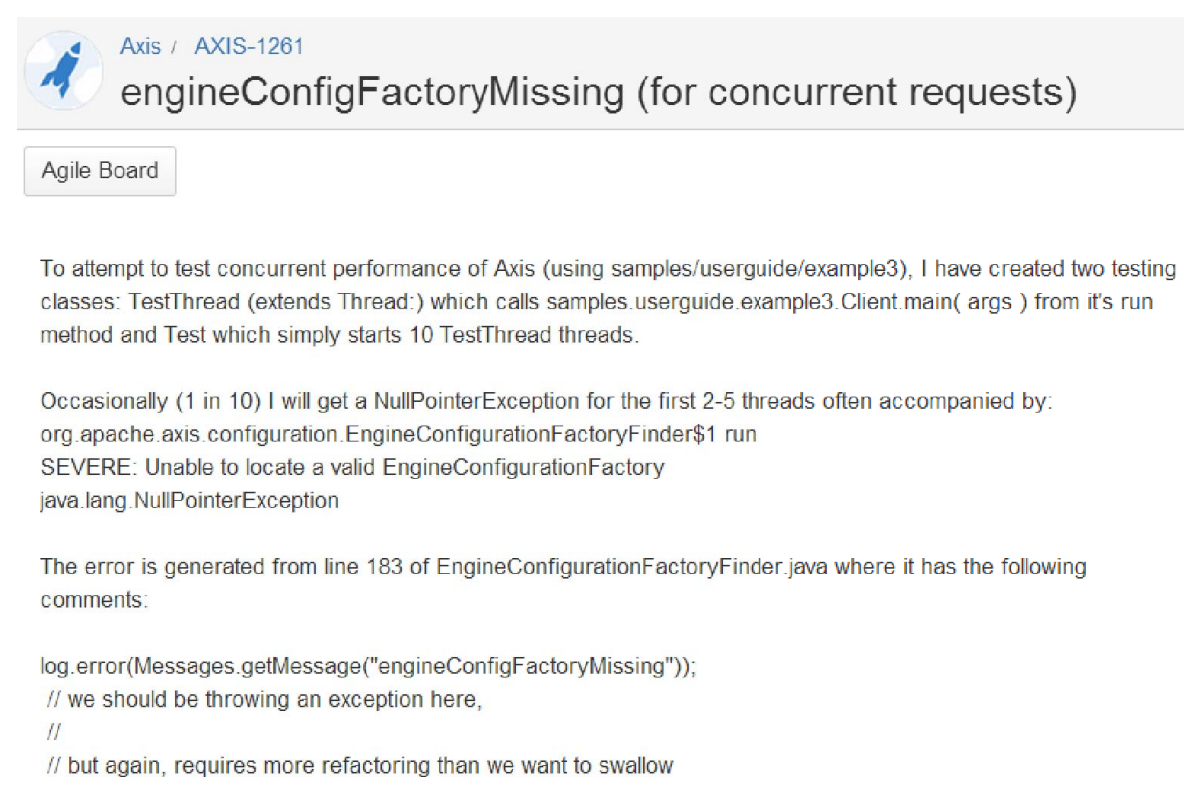

Fig. 2. An Example of Mandelbug in AXIS with BUGID=AXIS-1584.

to reproduce, and its fault trigging conditions are much more complex. Also previous study shows that Mandelbug needs more time to fix [6]. From the descriptions of defects in Figure 1 and 2, we notice that the defect in Figure 2 is much more difficult to fix, since it is a concurrency bug, and to reproduce the occurrence of the bug, developers need to collect more information.

2) Some terms in a bug report are good indicators to identify whether it is a Mandelbug, while some other terms are noise. For example, in Figure 2, the terms "concurrent", "occasionally" could help to identify a Mandelbug; while the terms "error", "test", and "class" are noise since both Bohrbug and Mandelbug are likely to use these terms when describing a defect. Thus, it is good to select good indicators (i.e., useful terms), and remove noise (i.e., useless terms) from the natural-language description of a defect.

The above observations tell us that we could use the natural-language description of defects to categorizes defects based on their fault triggering conditions, and selecting good terms (indicators) from defects could help to improve classification performance. Therefore, an automated tool which applies text mining techniques on the natural-language description of defects could assist developers to categorize defects. Based on these defect categories, developers can perform postmortem analysis and decide appropriate defect management strategies, e.g., refactoring of some components that are often affected by Mandelbugs.

\section{OVERALL FRAMEWORK}

Figure 3 shows our defect categorization framework. The whole framework includes two phases: model building phase and prediction phase. In the model building phase, our goal is to build a classifier (i.e., statistical model) by leveraging text mining techniques from historical bug reports with known labels (i.e., configuration or not). In the prediction phase, this classifier would be used to predict if an unknown bug report would be a Bohrbug or Mandelbug.

Our framework first extracts features from a set of training bug reports (i.e., bug reports with known status) (Step 1). Features are various quantifiable characteristics of bug reports that could potentially distinguish defects that are related to Mandelbugs from those are related Bohrbugs. The goal of feature extraction is to reduce the defects to some important, quantitative aspects. In this paper, we use textual features from the natural-language description of bug reports. Our framework extracts the texts from various fields (e.g., description, and summary fields) of bug reports. For each text, our framework tokenizes them, removes stop words (e.g., I, you, he, the), stems them (i.e., reduces them to their root forms, e.g., "configuration" and "configure" are reduced to "config"), and represents them in the form of a "bag of words" [10].

Then, our framework applies our fuzzy set based feature selection (USES) techniques to select a subset of relevant textual features to further improve the prediction performance (Step 2). ${ }^{5}$

After we select a subset of textual features, our framework next constructs a classifier (i.e., statistical model) based on the selected textual features of the training bug reports (Step 3). A classifier is a statistical model which assigns labels (in our case: Bohrbug or Mandelbug) to a data point (in our case: a defect) based on its textual features. The classifier construction phase would compare and contrast the features of bug reports that are Bohrbugs bugs, and those of bug reports that are Mandelbugs. Various classification algorithms can be used to build the classifier, e.g., naive Bayes multinomial [7], naive

${ }^{4}$ Detailed information of the feature extraction is presented in Section IV.

${ }^{5}$ Detailed information of USES is presented in Section V. 


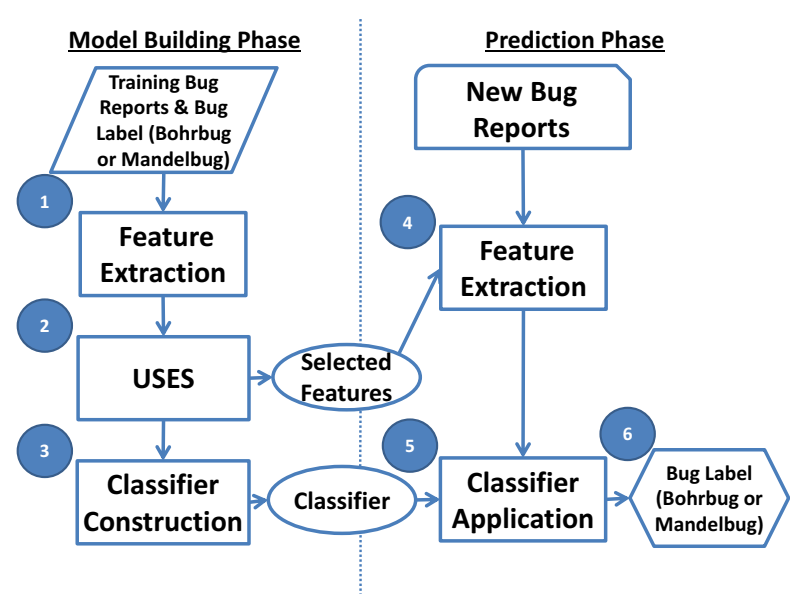

Fig. 3. Proposed Defect Categorization Framework.

Bayes [7], SVM [8], logistic regression [8], RBF network [9], and decision tree [8].

In the prediction phase, the classifier is then used to predict the categories of a defect with unknown label. For each of such bug reports, our framework first preprocesses and extracts textual features from it, and represents it by using the features selected in the model building phase (Step 4). Next, these features are input into the classifier in the classifier application step (Step 5). This step would output the prediction result which is one of the following labels: Bohrbug or Mandelbug (Step 6).

\section{Pre-Processing AND FeAtURe Extraction}

In this section, we first present our text pre-processing in Section IV-A. Then, we describe the textual feature extraction in Section IV-B.

\section{A. Text Pre-Processing}

We pro-process the natural-description of defects in 3 steps: tokenization, stop-word removal, and stemming.

1) Tokenization: Tokenization is the process that breaks a stream of text up into words, phrases, symbols, or other meaningful elements called tokens. We remove all numbers and punctuation marks appearing in the reports as they often have weak correlation with defect categories. We then extract the remaining word tokens.

2) Stop-word Removal: Stop words are words that are used often and carry little meaning to distinguish different categories of defects. Examples of stop word include "I", "you", "he", "the". We take a set of standard stop words from WVTool. ${ }^{6}$ These stop words are removed from the extracted word tokens.

3) Stemming: Stemming is the process to reduce inflected (or sometimes derived) words to their stem, base or root form. For example, the words "fishing", "fisher", and "fished" would all be reduced to "fish". We employ the well-known Porter stemmer ${ }^{7}$ to reduce a word to its representative root form.

\footnotetext{
${ }^{6}$ Available from: http://sourceforge.net/projects/wvtool/

${ }^{7}$ http://tartarus.org/Xmartin/PorterStemmer/
}

\section{B. Feature Extraction}

A typical bug report contains many fields. Some fields such as summary and description fields provide the naturallanguage description of a bug. The summary field is a condensed representation of a bug report, and the description field provides more detailed information. For example, Figures 1 and 2 present the summary and description fields of two bug reports in AXIS. In this paper, we extract features from the summary and description fields of bug reports.

We first perform text preprocessing strategies as described in Section IV-A. Next, we extract three kinds of textual features from these two bug report fields:

TEXT $T^{\text {Sum }}$ : We extract pre-processed word tokens from the summary field of a bug report as features. The value of each feature is the number of times the corresponding word token appears in the bug report.

$T E X T^{D e s c}$ : We extract pre-processed word tokens from the description field of a bug report as features. The value of each feature is the number of times the corresponding word token appears in the bug report.

$T E X T^{A l l}$ : We extract pre-processed word tokens from both summary and description fields of a bug report as features. The value of each feature is the number of times the corresponding word token appears in the bug report.

By default, we use TEXT $T^{A l l}$ as the features to construct a classifier, and we would also compare the performance of using TEXT $T^{\text {Sum }}$ and TEXT $T^{\text {Desc }}$ in Section VI.

\section{Fuzzy Set Based Feature Selection Method}

We use the processed word tokens as the features to construct a classifier. In total, we have a large number of word tokens. In machine learning literature, a feature can be viewed as a dimension, and a data point (i.e., a defect) can then be viewed as a point in a high-dimensional space. An overly high number of dimensions can cause the curse-ofdimensionality problem [8]. Aside from this, we notice for some datasets (e.g., apache HTTPD, and AXIS), only a very small minority of defects are Mandelbugs, i.e., class imbalance problem exists [11].

To address the above problems, in this section, we propose a new fuzzy set based feature selection method USES. We first present the definition of fuzzy category-word affinity score in Section V-A. Next, we present our feature selection method USES in Section V-B.

\section{A. Fuzzy Category-Word Affinity Score}

We denote the category of the $i^{t h}$ defects as $c_{i}$, and following vector space modeling [10], we represent the textual description of the $i^{t h}$ defect as a vector Defect $t_{i}=\left\langle t_{i, 1}, t_{i, 2}, \cdot \cdots\right.$ $\left.\cdot, t_{i, v}\right\rangle$, where $t_{i, j}$ is 1 if the word token $w_{j}$ appears in the $i^{t h}$ defects and 0 otherwise, and $v$ is the total number of unique terms in the whole defect collection. Based on these notations, we define fuzzy category-word affinity score as follows:

Definition 3: (Fuzzy Category-Word Affinity Score.) Consider a historical defect collection $D$, and a set of categories $C$. For each category $c \in C$, and word $w \in D$, the fuzzy 
category-word affinity score of $c$ and $w$, denoted as $\operatorname{Aff}(c, w)$, is computed as follows:

$$
\operatorname{Aff}(c, w)=\frac{n_{c, w}}{n_{c}+n_{w}-n_{c, w}}
$$

where $n_{c, w}$ denotes the number of defects whose descriptions contain word $w$ and are of category $c, n_{c}$ denotes the number of defects that are of category $c$, and $n_{w}$ denotes the number of defects that contain word $w$.

In our paper, we have two categories, Bohrbug and Mandelbug. We denote Bohrbug as $B$, and Mandelbug as $M$. Thus, for each word tokens $w \in D$, we have two fuzzy categoryword affinity scores, $\operatorname{Aff}(B, w)$, and $\operatorname{Aff}(M, w)$. We define the feature selection score of word $w$ as follows:

Definition 4: (Feature Selection Score.) For each word $w \in D$, its fuzzy Bohrbug-term affinity score and fuzzy Mandel-term affinity score are denoted as $\operatorname{Aff}(B, w)$, and $\operatorname{Aff}(M, w)$. The feature selection score of $\mathrm{w}$, denoted by Feature $(w)$, is the difference between these two scores, i.e.,

$$
\text { Feature }(w)=\operatorname{Aff}(M, w)-\operatorname{Aff}(B, w)
$$

Notice that the feature selection score for a word token $w$ could be a positive or a negative value. If a defects has many words with large positive feature selection scores then there is a high likelihood that it will be a Mandelbug. On the other hand, if a defect has many words with large negative feature selection scores, then there is a high likelihood that it is a Bohrbug.

Table I presents an example of a dataset with 4 words and 2 categories (Bohrbug (B) and Mandelbug (M)). We want to compute the fuzzy category-term affinity scores of word 1 , i.e., $\operatorname{Aff}(B, \operatorname{Word} 1)$, and $\operatorname{Aff}(M$, Word 1$)$. We notice that two defects (Defects 1 and 3) are Bohrbugs, i.e., $n_{B}=2$; Also, Word 1 appears in two defects (Defects 1 and 3), i.e., $n_{W \text { ord } 1}=2$. Furthermore, defects 1 and 3 both have Word 1 and are Bohrbugs, i.e., $n_{B, W \text { ord } 1}=2$. Thus, the fuzzy affinity score for Word1 and Bohrbug, denoted by $\operatorname{Aff}(B$, Word 1$)$, is

$$
\operatorname{Aff}(B, \text { Word } 1)=\frac{2}{2+2-2}=1
$$

Similarly, the fuzzy affinity score for word 1 and Mandelbug, i.e., $\operatorname{Aff}(M$, Word 1$)$, is

$$
\operatorname{Aff}(M, W \text { ord } 1)=\frac{0}{2+0-0}=0
$$

Finally, the feature selection score for Word 1, i.e., Feature (Word 1), is:

Feature $($ Word 1$)=\operatorname{Aff}(M, W$ ord 1$)-\operatorname{Aff}(B, W$ ord 1$)=-1$
TABLE I. AN EXAMPLE OF A DATASET WITH 4 WORDS AND 2 Categories (Bohrbug (B) and Mandelbug (M)). A Value in A CELl Is 1, IF The CORRESPONDING Word EXISTS IN THE DESCRIPTION of The CORRESPONDING DEFECT, AND IT Is 0 OTHERWISE.

\begin{tabular}{|c|c|c|c|c|c|}
\hline Defect ID & Word 1 & Word 2 & Word 3 & Word 4 & Category \\
\hline \hline 1 & 1 & 0 & 1 & 1 & B \\
\hline 2 & 0 & 1 & 0 & 1 & M \\
\hline 3 & 1 & 0 & 1 & 0 & B \\
\hline 4 & 0 & 1 & 0 & 0 & M \\
\hline
\end{tabular}

\section{1: $\mathbf{U S E S}(D, p \%, s p \%, I T E R)$}

2: Input:

3: $D$ : Training bug report collection

4: $p \%$ : Percentage of features to be selected

5: $s p \%$ : Percentage of training bug reports for classifier building

6: ITER: Number of iterations

7: Output: selectedFeatures

8: selectedFeatures: Final selected features (i.e., words)

9: Method:

10: Let $N=$ Total number of features (i.e., unique words) in $D$

11: Let the number of selected features $l=N \times p \%$;

12: Compute feature selection scores for each word in $D$

12: Compute feature selection scores for each word in $D$;
13: $\operatorname{candPos}=l$ words with the largest positive feature selection scores from $D$;

14: candNeg $=l$ words with the largest negative feature selection scores from $D$;

15: Merge candPos and candNeg into one set Merge.

16: Divide $D$ into two subsets $D_{\text {build }}$ and $D_{\text {validate }}$ according to $s p \%$;

17: Let bestFMeasure $=0$;

18: Let $i$ ter $=0$;

19: Let selectedFeatures $=\{\}$;

20: while iter $\leq I T E R$ do

21: Randomly select a subset of $l$ words $\left(T_{t m p}\right)$ from Merge; Randomly select a subset of $l$ words $\left(T_{t m p}\right)$ from Merge;
Build a classifier from $D_{b u i l d}$ based on the selected $l$ words;

Evaluate the Mandelbug F-measure $f_{t m p}$ of the classifier using $D_{\text {validate }}$

Evaluate the Mandelbug F-measure
if $f_{t m p}>$ best F Measure then

if $f_{\text {tmp }}>$ bestFMeasure
best F Measure $=f_{\text {tmp }}$

selectedFeatures $=T_{t m p}$

end if

iter $=i$ ter +1 ;

9: end while

0: Return selectedFeatures;

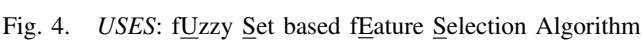

\section{B. USES}

Since there are many features and feature selection scores could be positive or negative, the selection of good features for effective classification is a challenging problem. One naive way is to select an equal number of features with the highest positive and negative scores. However, we propose USES that can perform better.

USES first selects $l$ features (i.e., words) with highest positive feature selection scores, and $l$ features with highest negative feature selection scores. In total, USES takes as input $2 l$ features. Then, it iterates many times. And in each iteration, it randomly selects a subset of $l$ features from the $2 l$ features, and a classifier is built on the $l$ features. We divide the training dataset into two subsets: one is used to select $l$ features, and another is used to evaluate the performance of the selected $l$ features. The classifier and the features with the best performance are selected. We set the value of $l$ to be high enough (i.e., $20 \%$ of the total number of features) so that a sufficient number of positive and negative word tokens are included.

Figure 4 presents our proposed method. First, we compute the number of features $l$ as $N \times p \%$, where $N$ is the total number of features (i.e., unique words) in a training bug report collection $D$ and $p$ is a parameter that decides the percentage of the features that would be selected. Then, we compute the feature selection scores for each word in $D$, and choose $l$ words 
with the largest positive feature selection scores (candPos) and $l$ words with the largest negative feature selection scores (candNeg), and merge these two sets (i.e., candPos and candNeg) into one set Merge (Lines 13, 14, and 15). Next, we divide the training set of bug reports $D$ into two subsets: $D_{\text {build }}$ and $D_{\text {validate }}$. We put $s p \%$ of the training bug reports into $D_{\text {build }}$ and the remaining into $D_{\text {validate }}$. By default, we put $90 \%$ of the defects in $D$ inside $D_{\text {build }}$, and the remaining $10 \%$ of the defects inside $D_{\text {validate }}$. Then, we iterate the process $I T E R$ times. By default, we set the number of iterations as 100 (i.e., ITER=100). For each iteration, we randomly select a subset of $l$ words (denoted as $T_{t m p}$ ) from Merge (Line 19). We train a classifier based on the selected features using $D_{\text {build }}$, and investigate the classifier's performance on $D_{\text {validate }}$, to evaluate how good a set of features (i.e., $T_{t m p}$ ) is. We record the Mandelbug F-measure that is achieved by the classifier. F-measure is a common measure to evaluate how good a classifier is. It is the harmonic mean of precision and recall. Precision refers to the proportion of defects predicted as Mandelbugs that are correctly predicted. Recall refers to the proportion of Mandelbugs that are correctly identified. We record the set $T_{t m p}$ that gives us the highest F-measure.

\section{EXPERIMENTS AND RESULTS}

In this section, we evaluate the effectiveness of our proposed tool. The experimental environment is an Intel(R) Core(TM) i5 $3.20 \mathrm{GHz}$ CPU, 4GB RAM desktop running Windows 7 (32-bit). We first present our experiment setup, evaluation metrics, and 4 research questions in Section VI-A, VI-B, and VI-C, respectively. We then present our experiment results that answer the four research questions (Sections VI-D, VI-E, VI-F, and VI-G).

\section{A. Experiment Setup}

We evaluate our proposed tool on 4 datasets from different open source software projects: Linux, Mysql, Apache HTTPD, and AXIS. These datasets were used by Cotroneo et al. in a previous empirical study [6]. Cotroneo et al. have manually analyzed hundreds of bug reports and classified each of them as a Bohrbug or a Mandelbug. For the Mysql dataset, we remove 7 duplicated defects from the original dataset by Cotroneo et al. Table II presents the statistics of the bug reports from the 4 projects. The columns correspond to the project name (Project), the number of bug reports collected (\# Bugs), the time period of the collected bug reports (Time), the number of Mandelbugs (\# M.), the number of Bohrbugs (\# B.), and the number of unique terms (\# Term). We remove terms which appear less than 5 times to reduce noise.

Stratified ten-fold cross validation [8] is used to evaluate the performance of our automated tool. We randomly divide the dataset into 10 folds. Of these 10 folds, 9 folds are use to train a classifier, while the last one fold is used to evaluate the performance of classifier. The whole process is iterated 10 times, and the average performance across the 10 iterations is recorded. Moreover, the distribution of labels in the training and test folds are the same as the original dataset to simulate the actual usage of our tool. Stratified cross validation is a standard evaluation setting, which is widely used in software engineering studies, c.f., [12]-[17].
TABle II. Statistics of Collected Datasets.

\begin{tabular}{|c|c|c|c|c|c|}
\hline Project & \#Bugs & Time & \#M. & \#B. & \#Term \\
\hline Linux & 267 & $2003.07-2011.05$ & 145 & 122 & 883 \\
\hline Mysql & 202 & $2006.08-2011.02$ & 78 & 124 & 515 \\
\hline HTTPD & 141 & $2002.03-2007.10$ & 25 & 116 & 376 \\
\hline AXIS & 199 & $2001.07-2005.11$ & 15 & 184 & 396 \\
\hline
\end{tabular}

TABLE IV. CONFUSION MATRIX.

\begin{tabular}{|c|c|c|}
\hline \multirow{2}{*}{ Classified as } & \multicolumn{2}{|c|}{ True Class } \\
\cline { 2 - 3 } & Mandelbug & Bohrbug \\
\hline Mandelbug & TP & FP \\
\hline Bohrbug & FN & TN \\
\hline
\end{tabular}

We implement USES on top of Weka [18]. ${ }^{8}$ By default, we set the number of selected words as $20 \%$ of the total number of words in the defects collections (i.e., $p \%=20 \%$ ). Also, we use naive Bayes multinomial as the classifier after we leverage USES to select words - we denote the combination of naive Bayes multinomial and USES as USES ${ }^{B}$.

\section{B. Evaluation Metrics}

To evaluate the predictive performance of our proposed tool, we create a confusion matrix to store prediction results. Table IV presents an example of a confusion matrix. The rows of the matrix correspond to predicted labels of defects. The columns of the matrix correspond to correct labels of defects. A cell in the matrix contains the number of bug reports of a particular predicted label and a particular correct label.

For each defects, there would be fours possible outcomes: a defect can be classified as a Mandelbug when it truly is a Mandelbug (true positive, TP); it can be classified as a Mandelbug when it is a Bohrbug (false positive, FP); it can be classified as a Bohrbug when it is a Mandelbug (false negative, FN); or it can be classified as a Bohrbug and it truly is a Mandelbug (true negative, TN). By using the values stored in the confusion matrix, in this paper, we calculate the accuracy, precision, recall and F-measure scores for each label (i.e., Mandelbug and Bohrbug) to evaluate the performance of our proposed tool.

Accuracy: the number of correctly classified defects (both Mandelbugs and Bohrbugs) over the total number of bugs, i.e., $A c c=\frac{T P+T N}{T P+F P+T N+F N}$.

Mandelbug Precision: the proportion of defects that are correctly labeled as Mandelbugs among those labeled as Mandelbugs, i.e., $P(C)=\frac{T P}{T P+F P}$.

Mandelbug Recall: the proportion of Mandelbugs bugs that are correctly labeled, i.e., $R(C)=\frac{T P}{T P+F N}$.

Bohrbug Precision: the proportion of bugs that are correctly labeled as Bohrbugs among those labeled as Bohrbugs, i.e., $P(N C)=\frac{T N}{T N+F N}$.

Bohrbug Recall: the proportion of Bohrbugs that are correctly labeled, i.e., $R(N C)=\frac{T N}{T N+F P}$.

F-measure: a summary measure that combines both precision and recall - it evaluates if an increase in precision (recall) outweighs a reduction in recall (precision). For Mandelbug F-measure, it is $F(C)=\frac{2 * P(C) * R(C)}{P(C)+R(C)}$. And for Bohrbug Fmeasure, it is $F(N C)=\frac{2 * P(N C) * R(N C)}{P(N C)+R(N C)}$.

\footnotetext{
${ }^{8} \mathrm{http} / / / w w w . c s . w a i k a t o . a c . n z / \mathrm{ml} /$ weka/
} 
Table III. Mandelbug F-measure, Bohrbug F-measure, and Accuracy for $U S E S^{B}$, Naive Bayes Multinomial (NBM), Naive Bayes (NB), SVM, Logistic Regression (LR), AND RBF NeTwork (RBF). The Last Column Shows the AVERAge Mandelbug F-MEasure, Bohrbug F-MeAsure, AND ACCURACY Scores ACross the 4 Datasets.

\begin{tabular}{|c|c|c|c|c|c|c|}
\hline Evaluation & Techniques & Linux & Mysql & HTTPD & Axis & Average \\
\hline \hline \multirow{4}{*}{ Mandelbug F-measure } & $U S E S^{B}$ & $\mathbf{0 . 5 2 4}$ & $\mathbf{0 . 6 1 5}$ & $\mathbf{0 . 3 7 5}$ & $\mathbf{0 . 2 9 8}$ & $\mathbf{0 . 4 5 3}$ \\
\cline { 2 - 7 } & $\mathrm{NBM}$ & $\mathbf{0 . 4 2 7}$ & $\mathbf{0 . 6 0 5}$ & $\mathbf{0 . 3 0 4}$ & $\mathbf{0 . 2 6 1}$ & $\mathbf{0 . 3 9 9}$ \\
\cline { 2 - 7 } & $\mathrm{NB}$ & 0.427 & 0.538 & 0.393 & 0.273 & 0.408 \\
\cline { 2 - 7 } & $\mathrm{SVM}$ & 0.695 & 0.196 & 0 & 0 & 0.223 \\
\cline { 2 - 7 } & $\mathrm{LP}$ & 0.502 & 0.497 & 0.391 & 0.054 & 0.361 \\
\cline { 2 - 7 } & $\mathrm{RBF}$ & 0.535 & 0.538 & 0.333 & 0.174 & 0.395 \\
\hline \hline \multirow{5}{*}{ Bohrbug F-measure } & $U S E S^{B}$ & $\mathbf{0 . 5 8 7}$ & $\mathbf{0 . 7 5 8}$ & $\mathbf{0 . 8 7 2}$ & $\mathbf{0 . 9 0 6}$ & $\mathbf{0 . 7 8 1}$ \\
\cline { 2 - 7 } & $\mathrm{NBM}$ & $\mathbf{0 . 5 9 9}$ & $\mathbf{0 . 7 6 2}$ & $\mathbf{0 . 8 6 4}$ & $\mathbf{0 . 9 0 3}$ & $\mathbf{0 . 7 8 2}$ \\
\cline { 2 - 7 } & $\mathrm{NB}$ & 0.599 & 0.710 & 0.833 & 0.910 & 0.763 \\
\cline { 2 - 7 } & $\mathrm{SVM}$ & 0.090 & 0.763 & 0.903 & 0.961 & 0.679 \\
\cline { 2 - 7 } & $\mathrm{LP}$ & 0.502 & 0.693 & 0.881 & 0.903 & 0.745 \\
\cline { 2 - 7 } & $\mathrm{RBF}$ & 0.528 & 0.741 & 0.863 & 0.949 & 0.770 \\
\hline \hline \multirow{5}{*}{ Accuracy } & $U S E S^{B}$ & $\mathbf{5 5 . 8 \%}$ & $\mathbf{7 0 . 3 \%}$ & $\mathbf{7 8 . 7 \%}$ & $\mathbf{8 3 . 4 \%}$ & $\mathbf{7 2 . 1 \%}$ \\
\cline { 2 - 7 } & $\mathrm{NBM}$ & $\mathbf{5 2 . 8 \%}$ & $\mathbf{7 0 . 3 \%}$ & $\mathbf{7 7 . 3 \%}$ & $\mathbf{8 2 . 9 \%}$ & $\mathbf{7 0 . 8 \%}$ \\
\cline { 2 - 7 } & $\mathrm{NB}$ & $52.8 \%$ & $64.4 \%$ & $73.8 \%$ & $83.9 \%$ & $68.7 \%$ \\
\cline { 2 - 7 } & $\mathrm{SVM}$ & $54.3 \%$ & $63.4 \%$ & $82.3 \%$ & $92.5 \%$ & $73.1 \%$ \\
\cline { 2 - 7 } & $\mathrm{LP}$ & $50.2 \%$ & $61.9 \%$ & $80.1 \%$ & $82.4 \%$ & $68.7 \%$ \\
\cline { 2 - 7 } & $\mathrm{RBF}$ & $55.8 \%$ & $60.9 \%$ & $83.0 \%$ & $92.0 \%$ & $71.9 \%$ \\
\hline
\end{tabular}

Notice that precision and recall are both important metrics for defect categorization since they measure quality of our tool in two aspects. If the precision is low, then the developer would not use the tool, due to a high number of false positives. If the recall is low, developers would not use the tool also, since most Mandelbugs (Bohrbugs) are not successfully predicted. There is a trade off between precision and recall, and one can increase precision by sacrificing recall (and vice versa) [8]. F-measure, which is the harmonic mean of precision and recall, is often used to judge whether an increase in precision outweighs a loss in recall (and vice versa) [8]. In many past papers in software engineering literature, e.g., [15], [19]-[21], F-measure is often used as a summary measure. Moreover, since Mandelbugs are more complex and require more time to be fixed [6], Mandelbug F-measure is the most important evaluation metric.

\section{Research Questions} tions:

We are interested to answer the following research ques-

RQ1 How effective is our proposed tool? How much improvement could our proposed tool achieve over vanilla classification techniques without any feature selection?

In our proposed tool, we first use USES to select features, and then we build a classifier based on the selected features. We would like to investigate whether our proposed tool could achieve better performance over standard classification techniques without any feature selection. Answer to this research question would shed light to whether our proposed tool advances existing classification methods. To answer this research question, we select 5 state-of-the-art classification techniques, i.e., naive Bayes multinomial [7], naive Bayes [7], SVM [8], logistic regression [8], and RBF network [9]. For SVM, we use its libSVM implementation [22]. For the other techniques, we use their implementations in Weka. We compare USES with these techniques.

RQ2 Can USES achieve a better performance over other feature selection techniques such as information gain, gain ratio, and one rule [8]?

In data mining literature, there are many feature selection techniques. Information gain, gain ratio, and one rule based approaches are popular feature selection techniques [8]. We want to investigate whether USES could achieve a better performance over information gain (IG), gain ratio (GR), and one rule (OneR). We first select features using USES, IG, GR, and OneR, and then for each resulting feature set, we create a classifier. We compare the effectiveness of these classifiers to demonstrate whether USES is better than the other feature selection techniques.

RQ3 What are the impact of using various textual features on the effectiveness of our proposed approach?

Based on the description in Section IV-B, we can extract 3 types of feature sets from bug reports, i.e., Text $t^{\text {Sum }}$, Text ${ }^{\text {Desc }}$, Text ${ }^{A l l}$. We would like to investigate the effectiveness of these 3 feature sets to categorize defects. To answer this research question, we build 3 classifiers each using one of these 3 feature sets, and evaluate the performance of these 3 classifiers.

RQ4 Do different numbers of selected features affect the performance of our proposed tool?

By default, USES selects $20 \%$ of all textual features. We investigate whether different numbers of selected features (i.e., words) would affect the performance of our proposed tool. To answer this research question, we vary the number of selected features from $5 \%, 10 \%, 15 \%, 20 \%, 25 \%$ to $30 \%$ of the total number of features.

\section{RQ1: Performance of Our Tool}

Table III presents the Mandelbug F-measure, Bohrbug Fmeasure, and accuracy for $U S E S^{B}$ compared with naive Bayes multinomial, naive Bayes, SVM, logistic regression, and RBF network. The Mandelbug F-measure, Bohrbug F-measure, and accuracy for $U S E S^{B}$ vary from $0.298-0.615,0.587$ - 0.906 , and $55.8 \%-83.4 \%$, respectively. Considering all five other classification techniques, we notice that naive Bayes multinomial achieves the best performance. Our proposed $U S E S^{B}$ improves the Mandelbug F-measure of naive Bayes multinomial by $22.7 \%, 1.7 \%, 23.4 \%$, and $14.2 \%$ for Linux, Mysql, Apache HTTPD, and Axis, respectively. Averaging across the 4 datasets, the average improvement achieved by $U S E S^{B}$ is $15.48 \%$. 
table V. Our Proposed Tool USES with Naive Bayes Multinomial(NBM) Vs. Information Gain with NBM (IG).

\begin{tabular}{|c|c|c|c|c|c|c|}
\hline Evaluation & Techniques & Linux & Mysql & HTTPD & Axis & Average. \\
\hline \hline \multirow{3}{*}{ Mandelbug F-measure } & $U S E S^{B}$ & 0.524 & 0.615 & 0.375 & 0.298 & 0.453 \\
\cline { 2 - 7 } & IG & 0.486 & 0.532 & 0.308 & 0.286 & 0.403 \\
\cline { 2 - 7 } & Impro. & $\mathbf{7 . 8 \%}$ & $\mathbf{1 5 . 6 \%}$ & $\mathbf{2 1 . 8 \%}$ & $\mathbf{4 . 2 \%}$ & $\mathbf{1 2 . 3 \%}$ \\
\hline \hline \multirow{3}{*}{ Bohrbug F-measure } & $U S E S^{B}$ & 0.587 & 0.758 & 0.872 & 0.906 & 0.781 \\
\cline { 2 - 7 } & IG & 0.557 & 0.699 & 0.843 & 0.916 & 0.754 \\
\cline { 2 - 7 } & Impro. & $\mathbf{5 . 4 \%}$ & $\mathbf{8 . 4 \%}$ & $\mathbf{3 . 4 \%}$ & $\mathbf{- 1 . 1 \%}$ & $\mathbf{4 . 0 \%}$ \\
\hline \hline \multirow{3}{*}{ Accuracy } & $U S E S^{B}$ & $55.8 \%$ & $70.3 \%$ & $78.7 \%$ & $83.4 \%$ & $72.1 \%$ \\
\cline { 2 - 7 } & IG & $52.4 \%$ & $63.4 \%$ & $74.5 \%$ & $84.9 \%$ & $68.8 \%$ \\
\cline { 2 - 7 } & Impro. & $\mathbf{6 . 4 \%}$ & $\mathbf{1 0 . 9 \%}$ & $\mathbf{5 . 7 \%}$ & $\mathbf{- 1 . 8 \%}$ & $\mathbf{5 . 3 \%}$ \\
\hline
\end{tabular}

Table VI. Our Proposed Tool USES with Naive Bayes Multinomial(NBM) VS. Gain Ratio With NBM (GR).

\begin{tabular}{|c|c|c|c|c|c|c|}
\hline Evaluation & Techniques & Linux & Mysql & HTTPD & Axis & Average. \\
\hline \hline \multirow{3}{*}{ Mandelbug F-measure } & $U S E S^{B}$ & 0.524 & 0.615 & 0.375 & 0.298 & 0.453 \\
\cline { 2 - 7 } & GR & 0.486 & 0.532 & 0.302 & 0.286 & 0.402 \\
\cline { 2 - 7 } & Impro. & $\mathbf{7 . 8 \%}$ & $\mathbf{1 5 . 6 \%}$ & $\mathbf{2 4 . 2 \%}$ & $\mathbf{4 . 2 \%}$ & $\mathbf{1 2 . 9 \%}$ \\
\hline \hline \multirow{3}{*}{ Bohrbug F-measure } & $U S E S^{B}$ & 0.587 & 0.758 & 0.872 & 0.906 & 0.781 \\
\cline { 2 - 7 } & GR & 0.557 & 0.699 & 0.838 & 0.916 & 0.753 \\
\cline { 2 - 7 } & Impro. & $\mathbf{5 . 4 \%}$ & $\mathbf{8 . 4 \%}$ & $\mathbf{4 . 1 \%}$ & $\mathbf{- 1 . 1 \%}$ & $\mathbf{4 . 2 \%}$ \\
\hline \hline \multirow{2}{*}{ Accuracy } & $U S E S^{B}$ & $55.8 \%$ & $70.3 \%$ & $78.7 \%$ & $83.4 \%$ & $72.1 \%$ \\
\cline { 2 - 7 } & GR & $52.4 \%$ & $63.4 \%$ & $74.5 \%$ & $84.9 \%$ & $68.8 \%$ \\
\cline { 2 - 7 } & Impro. & $\mathbf{6 . 4 \%}$ & $\mathbf{1 0 . 9 \%}$ & $\mathbf{5 . 7 \%}$ & $\mathbf{- 1 . 8 \%}$ & $\mathbf{5 . 3 \%}$ \\
\hline
\end{tabular}

We notice that for SVM, its Mandelbug F-measure for Linux is quite high, i.e., 0.695. However, considering its Bohrbug F-measure for Linux, we find that SVM predicts nearly every defect as Mandelbug, since in Linux, Mandelbugs are the majority. For Apache HTTPD and Axis, SVM also nearly predicts every defect as Bohrbug, since Bohrbugs are the majority. Thus, although the average accuracy of SVM is higher than $U S E S^{B}$, it does not mean that SVM performs better.

\section{E. RQ2: USES vs. IG, GR, and OneR}

Table $\mathrm{V}$ presents the experiment results of our $U S E S^{B}$ compared to information gain with naive Bayes multinomial (IG). We notice that the differences in F-measures and accuracy are substantial. $U S E S^{B}$ improves the average Mandelbug Fmeasure, Bohrbug F-measure, and accuracy of IG by $12.3 \%$, $4.0 \%$, and $5.3 \%$, respectively. Table VI presents the experiment results of our $U S E S^{B}$ compared to gain ratio with naive Bayes multinomial (GR). We notice that the differences in F-measures and accuracy are substantial. On average across the 4 datasets, $U S E S^{B}$ improves the average Mandelbug Fmeasure, Bohrbug F-measure, and accuracy of GR by $12.9 \%$, $4.2 \%$, and $5.3 \%$, respectively. Table VII presents the experiment results of our $U S E S^{B}$ compared to one rule with naive Bayes multinomial (OneR). We notice that the differences in F-measures and accuracy are substantial. On average across the 4 datasets, $U S E S^{B}$ improves the average Mandelbug F-measure, Bohrbug F-measure, and accuracy of OneR by $68.3 \%, 4.2 \%$, and $4.6 \%$, respectively.

\section{F. RQ3: Effect of Various Textual Features}

Table VIII presents the experiment results of using various textual features, i.e., Text $t^{A l l}$, Text $t^{\text {Desc }}$, and Text ${ }^{\text {Sum }}$. We notice that Text ${ }^{A l l}$ achieves the best performance as compared to Text ${ }^{\text {Desc }}$, and Text ${ }^{\text {Sum }}$.

\section{G. RQ4: Effect of Varying the Number of Selected Features}

Figure 5 presents the Mandelbug F-measure of $U S E S^{B}$ for various numbers of selected features (i.e., words) for

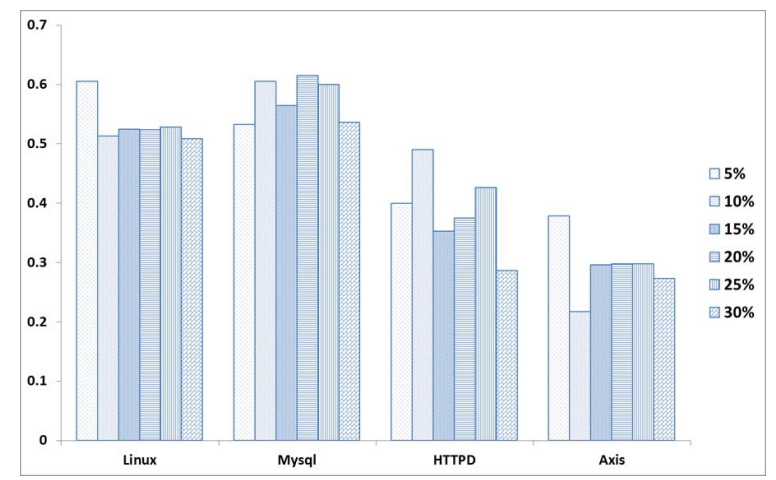

Fig. 5. Mandelbug F-measures for Different Numbers of Selected Features ( $5 \%$ to $30 \%$ of the Number of Distinct Words in the Training Data).

Linux, Mysql, Apache HTTPD, and Axis. Notice that for small numbers of features (e.g., $5 \%, 10 \%$, and $15 \%$ of the number of distinct words), the Mandelbug F-measure scores are unstable. For example, for Apache HTTPD, its Mandelbug F-measure is 0.4 when $5 \%$ of the number of distinct words are selected, it becomes 0.49 when $10 \%$ of the number of distinct words are selected, and it becomes 0.353 when $15 \%$ of the number of distinct words are selected. For a larger number of features (e.g., 20\%, 25\%, and 30\% of the number of distinct words), the Mandelbug F-measure scores are more stable. For example, for Axis, its Mandelbug F-measure is 0.298 when $20 \%$ of the number of distinct words are selected, it becomes 0.298 when $25 \%$ of the number of distinct words are selected, and it becomes 0.273 when $30 \%$ of the number of distinct words are selected.

\section{THREATS TO VALIDITY}

Threats to internal validity relate to errors in our experiments. We use the same datasets provide by Cotroneo et al. [6]. We have double checked our experiments and removed the duplicated defects from the datasets, still there could be errors that we do not notice. 
Table ViI. Our Proposed Tool USES with Naive Bayes Multinomial(NBM) VS. One Rule with NBM (OneR).

\begin{tabular}{|c|c|c|c|c|c|c|}
\hline Evaluation & Techniques & Linux & Mysql & HTTPD & Axis & Average. \\
\hline \hline \multirow{3}{*}{ Mandelbug F-measure } & $U S E S^{B}$ & 0.524 & 0.615 & 0.375 & 0.298 & 0.453 \\
\cline { 2 - 7 } & OneR & 0.498 & 0.547 & 0.302 & 0.293 & 0.091 \\
\cline { 2 - 7 } & Impro. & $\mathbf{5 . 2 \%}$ & $\mathbf{1 2 . 4 \%}$ & $\mathbf{2 8 . 0 \%}$ & $\mathbf{2 2 7 . 5 \%}$ & $\mathbf{6 8 . 3 \%}$ \\
\hline \hline \multirow{3}{*}{ Bohrbug F-measure } & $U S E S^{B}$ & 0.587 & 0.758 & 0.872 & 0.906 & 0.781 \\
\cline { 2 - 7 } & OneR & 0.548 & 0.7 & 0.88 & 0.887 & 0.754 \\
\cline { 2 - 7 } & Impro. & $\mathbf{7 . 1 \%}$ & $\mathbf{8 . 3 \%}$ & $\mathbf{- 0 . 9 \%}$ & $\mathbf{2 . 1 \%}$ & $\mathbf{4 . 2 \%}$ \\
\hline \hline \multirow{3}{*}{ Accuracy } & $U S E S^{B}$ & $55.8 \%$ & $70.3 \%$ & $78.7 \%$ & $83.4 \%$ & $72.1 \%$ \\
\cline { 2 - 7 } & OneR & $52.4 \%$ & $63.9 \%$ & $79.4 \%$ & $80.0 \%$ & $68.9 \%$ \\
\cline { 2 - 7 } & Impro. & $\mathbf{6 . 4 \%}$ & $\mathbf{1 0 . 1 \%}$ & $\mathbf{- 0 . 9 \%}$ & $\mathbf{4 . 4 \%}$ & $\mathbf{4 . 6 \%}$ \\
\hline
\end{tabular}

TABLE VIII. VARIOUS TEXT FEATURES USED For ClassificATION.

\begin{tabular}{|c|c|c|c|c|c|c|}
\hline Evaluation & Features & Linux & Mysql & HTTPD & Axis & Average. \\
\hline \multirow{3}{*}{ Mandelbug F-measure } & Text ${ }^{A l l}$ & 0.524 & 0.615 & 0.375 & 0.298 & 0.453 \\
\hline & Text $t^{\text {Desc }}$ & 0.478 & 0.595 & 0.383 & 0.292 & 0.437 \\
\hline & Text ${ }^{\text {Sum }}$ & 0.690 & 0.383 & 0.200 & 0.00 & 0.318 \\
\hline \multirow{3}{*}{ Bohrbug F-measure } & Text ${ }^{A l t}$ & 0.587 & 0.758 & 0.872 & 0.906 & 0.781 \\
\hline & Text ${ }^{\text {Desc }}$ & 0.551 & 0.740 & 0.877 & 0.903 & 0.768 \\
\hline & Text ${ }^{\text {Sum }}$ & 0.313 & 0.754 & 0.905 & 0.961 & 0.733 \\
\hline \multirow{3}{*}{ Accuracy } & $\overline{T e x t^{A l l}}$ & $55.8 \%$ & $70.3 \%$ & $78.7 \%$ & $83.4 \%$ & $72.1 \%$ \\
\hline & Text ${ }^{\text {Desc }}$ & $51.7 \%$ & $68.3 \%$ & $79.4 \%$ & $82.9 \%$ & $70.6 \%$ \\
\hline & Text ${ }^{\text {Sum }}$ & $57.3 \%$ & $64.9 \%$ & $83.0 \%$ & $92.5 \%$ & $74.4 \%$ \\
\hline
\end{tabular}

Threats to external validity relate to the generalizability of our results. We have analyzed 809 bug reports from 4 open source software projects. In the future, we plan to reduce this threat further by analyzing more bug reports from open source and commercial software projects .

Threats to construct validity refer to the suitability of our evaluation metrics. We use Mandelbug F-measure as the main evaluation metric. F-measure has also been used by past software engineering studies to evaluate the effectiveness of a prediction technique [15], [19]-[21]. Thus, we believe there is little threat to construct validity.

\section{RELATED WORK}

In this section, we discuss some related works on characterizing or predicting the types of bugs in Section VIII-A. Next, we briefly review some works on text mining for software engineering in Section VIII-B.

\section{A. Characterization and Prediction of Bugs}

There have been a number of studies on characterizing or predicting the types of bugs [6], [23]-[27]. Gegick et al. propose the usage of text mining techniques to identify whether a bug is a security bug or not [23]. Zaman et al. perform an empirical study on security bugs and performance bugs in Firefox [25]. They find security bugs need more time to be fixed, while performance bugs are not that different from other bugs, in terms of bug fix time, but more files need to be changed to fix them. Arshad et al. extract configuration bugs from GlassFish and JBoss, and they characterize configuration bugs from several dimensions, i.e., problem-type, problemtime, problem-manifestation, and problem-culprit [24]. Based on their findings, they also develop a tool named ConfGauge which injects parameter-based configuration issues into software systems. Cotroneo et al. perform an empirical study on Borhbug and Mandelbug in open-source software projects, and they conclude that Mandelbugs need longer time to fix, and require specific strategies to be dealt with [6]. Xia et al. perform an empirical study on bugs in software build systems such as Ant, Maven, CMake and QMake, and they find that $21.35 \%$ of the build system bugs are related to external interface problems [26]. Thung et al. propose a method to automatically categorize bug reports into 3 families: control and data flow, structural, and non-functional [27]. Xia et al. propose the usage of data mining and feature selection techniques to identify configuration bugs [28]. Our work complements the above studies; we classify a bug as a Bohrbug or a Mandelbug.

\section{B. Text Mining for Software Engineering}

There have been a number of studies on text mining for software engineering [20], [29]-[33]. The survey here is by no means complete. Sun et al. propose a text mining technique and extend BM25F to accurately detect duplicated bug reports [29], [30]. Later, Nguyen et al. combine topic model and BM25F to achieve a better performance in detecting duplicated bug reports [31]. Wu et al. propose Relink which leverages information retrieval techniques to recover links between bugs and their corresponding changes [20]. Marcus and Maletic use Latent Semantic Indexing (LSI) to recover traceability links between documentation to source code [32]. Zhou et al. propose an approach that takes in a bug report and return source code files that are likely to be relevant to the input bug report [34]. Haiduc et al. use automated text summarization to produce succinct and informative text to comprehend software code [33]

\section{CONCLUSION AND Future WORK}

In this paper, we propose a text mining solution which categorizes defects into fault trigger categories by analyzing the natural-language description of bug reports. Considering Mandelbugs are more complex, and need more time to fix, we propose a novel fuzzy set based feature selection algorithm named USES, which selects the features (i.e., terms) which have high ability to distinguish Mandelbugs from Bohrbugs. USES first caches a set of terms based on their fuzzy affinity scores to Bohrbug or Mandelbug. Next, it iterates many times, and in each iteration, it selects a subset of terms, builds a classifier on these terms, and evaluates it using a subset of a training data. At the end of the iterations, USES selects 
the classifier and the terms which could achieve the best Mandelbug F-measure scores on thetraining data. We evaluate our solution on 4 datasets including Linux, Mysql, Apache HTTPD, and AXIS containing a total of 809 bug reports. We show that USES with naive Bayes multinomial achieves the best performance over many baseline approaches. On average across the 4 projects, USES improves Mandelbug F-measure scores of information gain with naive Bayes multinomial, which is the best performing baseline, by $12.3 \%$.

In the future, we plan to evaluate our proposed tool with more defects from more software projects, and develop a better technique which could further improve the performance of defect categorization based on fault triggering conditions. We also plan to build a model leveraging transfer learning [35] to predict Mandelbugs by using data from other projects.

\section{ACKNOWLEDGMENT}

This research is sponsored in part by NSFC Program (No.61103032) and National Key Technology R\&D Program of the Ministry of Science and Technology of China (2014BAH24F02).

\section{REFERENCES}

[1] J. S. Collofello and S. N. Woodfield, "Evaluating the effectiveness of reliability-assurance techniques," Journal of systems and software, vol. 9, no. 3, pp. 191-195, 1989.

[2] Z. Li, L. Tan, X. Wang, S. Lu, Y. Zhou, and C. Zhai, "Have things changed now?: an empirical study of bug characteristics in modern open source software," in Proceedings of the 1st workshop on Architectural and system support for improving software dependability, 2006.

[3] S. Lu, S. Park, E. Seo, and Y. Zhou, "Learning from mistakes: a comprehensive study on real world concurrency bug characteristics," in ACM Sigplan Notices, vol. 43, no. 3. ACM, 2008, pp. 329-339.

[4] J. Gray, "Why do computers stop and what can be done about it?" in Symposium on reliability in distributed software and database systems. Los Angeles, CA, USA, 1986, pp. 3-12.

[5] M. Grottke and K. S. Trivedi, "A classification of software faults," Journal of Reliability Engineering Association of Japan, 2005.

[6] D. Cotroneo, M. Grottke, R. Natella, R. Pietrantuono, and K. S. Trivedi, "Fault triggers in open-source software: An experience report," in Software Reliability Engineering (ISSRE), 2013 IEEE 24th International Symposium on. IEEE, 2013, pp. 178-187.

[7] A. McCallum, K. Nigam et al., "A comparison of event models for naive bayes text classification," in AAAI-98 workshop on learning for text categorization, vol. 752. Citeseer, 1998, pp. 41-48.

[8] J. Han, M. Kamber, and J. Pei, Data mining: concepts and techniques. Morgan kaufmann, 2006

[9] M. J. Orr et al., "Introduction to radial basis function networks," 1996.

[10] R. Baeza-Yates, B. Ribeiro-Neto et al., Modern information retrieval. ACM press New York, 1999, vol. 463.

[11] H. He and E. A. Garcia, "Learning from imbalanced data," Knowledge and Data Engineering, IEEE Transactions on, vol. 21, no. 9, pp. $1263-$ 1284, 2009.

[12] E. Shihab, A. Ihara, Y. Kamei, W. M. Ibrahim, M. Ohira, B. Adams, A. E. Hassan, and K.-i. Matsumoto, "Predicting re-opened bugs: A case study on the eclipse project," in Reverse Engineering (WCRE), 2010 17th Working Conference on. IEEE, 2010, pp. 249-258.

[13] X. Xia, Y. Feng, D. Lo, Z. Chen, and X. Wang, "Towards more accurate multi-label software behavior learning," in Software Maintenance, Reengineering and Reverse Engineering (CSMR-WCRE), 2014 Software Evolution Week-IEEE Conference on. IEEE, 2014, pp. 134-143.

[14] X. Xia, D. Lo, X. Wang, and B. Zhou, "Tag recommendation in software information sites," in Proceedings of the Tenth International Workshop on Mining Software Repositories. IEEE Press, 2013, pp. 287-296.
[15] Y. Tian, J. Lawall, and D. Lo, "Identifying linux bug fixing patches," in Software Engineering (ICSE), 2012 34th International Conference on. IEEE, 2012, pp. 386-396.

[16] T. Menzies and A. Marcus, "Automated severity assessment of software defect reports," in Software Maintenance, 2008 ICSM 2008. IEEE International Conference on. IEEE, 2008, pp. 346-355.

[17] F. Thung, D. Lo, and J. L. Lawall, "Automated library recommendation," in WCRE, 2013, pp. 182-191.

[18] M. Hall, E. Frank, G. Holmes, B. Pfahringer, P. Reutemann, and I. H. Witten, "The weka data mining software: an update" ACM SIGKDD Explorations Newsletter, vol. 11, no. 1, pp. 10-18, 2009.

[19] A. T. Nguyen, T. T. Nguyen, H. A. Nguyen, and T. N. Nguyen, "Multilayered approach for recovering links between bug reports and fixes," in Proceedings of the ACM SIGSOFT 20th International Symposium on the Foundations of Software Engineering. ACM, 2012, p. 63.

[20] R. Wu, H. Zhang, S. Kim, and S.-C. Cheung, "Relink: recovering links between bugs and changes." in SIGSOFT FSE, 2011, pp. 15-25.

[21] J. Nam, S. J. Pan, and S. Kim, "Transfer defect learning," in Proceedings of the 2013 International Conference on Software Engineering, 2013.

[22] C.-C. Chang and C.-J. Lin, "Libsvm: a library for support vector machines," ACM Transactions on Intelligent Systems and Technology (TIST), vol. 2, no. 3, p. 27, 2011.

[23] M. Gegick, P. Rotella, and T. Xie, "Identifying security bug reports via text mining: An industrial case study," in Mining Software Repositories (MSR), 2010 7th IEEE Working Conference on. IEEE, 2010, pp. 11-20.

[24] F. A. Arshad, R. J. Krause, and S. Bagchi, "Characterizing configuration problems in java ee application servers: An empirical study with glassfish and jboss," pp. 198-207, 2013.

[25] S. Zaman, B. Adams, and A. E. Hassan, "Security versus performance bugs: a case study on firefox," in Proceedings of the 8th working conference on mining software repositories. ACM, 2011, pp. 93-102.

[26] X. Xia, X. Zhou, D. Lo, and X. Zhao, "An empirical study of bugs in software build systems," in Quality Software (QSIC), 2013 13th International Conference on. IEEE, 2013, pp. 200-203.

[27] F. Thung, D. Lo, and L. Jiang, "Automatic defect categorization," in Reverse Engineering (WCRE), 2012 19th Working Conference on. IEEE, 2012, pp. 205-214.

[28] X. Xia, D. Lo, W. Qiu, X. Wang, and B. Zhou, "Automated configuration bug report prediction using text mining," in 38th Annual International Computers, Software \& Applications Conference (COMPSAC). IEEE, 2014.

[29] C. Sun, D. Lo, X. Wang, J. Jiang, and S.-C. Khoo, "A discriminative model approach for accurate duplicate bug report retrieval," in Proceedings of the 32nd ACM/IEEE International Conference on Software Engineering-Volume 1. ACM, 2010, pp. 45-54.

[30] C. Sun, D. Lo, S.-C. Khoo, and J. Jiang, "Towards more accurate retrieval of duplicate bug reports," in Proceedings of the 2011 26th IEEE/ACM International Conference on Automated Software Engineering. IEEE Computer Society, 2011, pp. 253-262.

[31] A. T. Nguyen, T. T. Nguyen, T. N. Nguyen, D. Lo, and C. Sun, "Duplicate bug report detection with a combination of information retrieval and topic modeling," in Automated Software Engineering (ASE), 2012 Proceedings of the 27th IEEE/ACM International Conference on. IEEE, 2012, pp. 70-79.

[32] A. Marcus and J. I. Maletic, "Recovering documentation-to-sourcecode traceability links using latent semantic indexing," in Software Engineering, 2003. Proceedings. 25th International Conference on. IEEE, 2003, pp. 125-135.

[33] S. Haiduc, J. Aponte, and A. Marcus, "Supporting program comprehension with source code summarization," in Software Engineering, 2010 ACM/IEEE 32nd International Conference on, vol. 2. IEEE, 2010, pp. 223-226.

[34] J. Zhou, H. Zhang, and D. Lo, "Where should the bugs be fixed? more accurate information retrieval-based bug localization based on bug reports," in ICSE, 2012, pp. 14-24.

[35] S. J. Pan and Q. Yang, "A survey on transfer learning," Knowledge and Data Engineering, IEEE Transactions on, vol. 22, no. 10, pp. 13451359, 2010. 